\title{
Capacity Region of a Wireless Mesh Backhaul Network over the CSMA/CA MAC
}

\author{
Yu Cheng, Hongkun Li \\ Dept. Electrical \& Computer Engineering \\ Illinois Institute of Technology \\ \{cheng, hli55\}@iit.edu
}

\author{
Pengjun Wan \\ Dept. Computer Science \\ Illinois Institute of Technology \\ wan@cs.iit.edu
}

\author{
Xinbing Wang \\ Dept. Electronic Engineering \\ Shanghai Jiaotong University \\ xwang8@ @jtu.edu.cn
}

\begin{abstract}
This paper studies the maximum throughput that can be supported by a given wireless mesh backhaul network, over a practical CSMA/CA medium access control (MAC) protocol. We resort to the multi-commodity flow (MCF) formulation, augmented with the conflict-graph constraints, to jointly compute the maximum throughput and the associated optimal network dimensioning; while use a novel approach to take into account the collision overhead in the distributed CSMA/CA MAC. Such overhead has been ignored by the existing MCF-based capacity studies, which assume impractical centralized scheduling and result in aggressive network dimensioning, unachievable over the CSMA/CA MAC. We develop a generic method to integrate the CSMA/CA MAC analysis with the MCF formulation for optimal network capacity analysis, and derive both an upper bound and a lower bound of the network throughput over a practical CSMA/CA protocol. To the best of our knowledge, this paper is the first rigorous theoretical study of the achievable capacity over a multi-hop CSMA/CA based wireless network.
\end{abstract}

\section{INTRODUCTION}

The network capacity of importance to a multi-hop wireless backhaul network is the total throughput traversing the given set of ingress/egress edge nodes. The maximum network capacity is normally coupled with optimal routing and scheduling to form a network dimensioning issue [1], [2]. The conflict graph or contention graph is the popular tool to model the interference among different wireless links [3]-[5]. The mainthread approach for wireless network dimensioning is to apply a multi-commodity flow (MCF) formulation, augmented with constraints derived from the conflict graph [1]-[3], [6], [7].

The existing MCF-based dimensioning studies mainly focus on the independent set based formulation, where the MCF solution can generates an optimal centralized scheduling (under the assumption of a synchronized slotted system): the maximal independent sets take turns in grabbing the channel for data transmission, with the proportion of transmission time for each set determined by the MCF solution. However, the maximum throughput based on the optimal centralized scheduling is not achievable for a network applying a distributed carrier sensing multiple access with collision avoidance (CSMA/CA) protocol for medium access control (MAC), say, the IEEE 802.11

The work of Y. Cheng and P. Wan was supported in part by NSF grant CNS-0832093 and CNS-0916666, respectively.

The work of X. Wang was supported by grants NSF China 60702046 and 60832005 . distributed coordination function (DCF). The main reason is that collisions are inevitable for the CSMA/CA MAC due to its nature of random access, which leads to reduced channel utilization. It is of critical importance to develop analytical tools for determining the maximum backhaul throughput that is achievable over a practical CSMA/CA MAC.

In this paper, we develop a generic method to integrate CSMA/CA MAC analysis with the MCF formulation for optimal network capacity analysis by resorting to the clique-based method. A clique contains links that mutually conflict with each other. By considering each clique as one single-hop area, we reveal that applying the channel utilization analysis for a single-hop CSMA/CA area [8] to each clique gives a generic method to correlate the MCF formulation with the CSMA/CA MAC modeling, which readily leads to a tighter upper bound of the network throughput over the CSMA/CA MAC. Another contribution is a theoretical study of the relationship between a CSMA/CA area and its associated CSMA/CA clique. A CSMA/CA clique consists of the links incident to the nodes in the CSMA/CA area. The CSMA/CA clique is a newly proposed concept, different from the maximal clique. Note that the clique focuses on the interference among links, whereas a CSMA/CA area focuses on the interference among sending nodes in spite of the receiver nodes. We theoretically prove that the total normalized throughput over a CSMA/CA clique can be transformed to that over a CSMA/CA area. Such theoretical results provide a foundation for deriving the lower bound of the network throughput. The third main contribution is the theoretical proof of a sufficient condition that guarantees the achievability of a network flow allocation over the CSMA/CA MAC. Based on such a sufficient condition and the relationship between a CSMA/CA clique and a maximal clique, we then derive a lower bound of the network throughput. To the best of our knowledge, this paper is the first rigorous theoretical study of achievable capacity over a multi-hop CSMA/CA based wireless network.

The remainder of this paper is organized as follows. Section II reviews more related work. Section III summarizes the CSMA/CA optimal operation point analysis. Section IV describes the MCF formulation for wireless backhaul networks. Section $\mathrm{V}$ investigates the upper bound and lower bound of the network capacity over the CSMA/CA MAC. Section VI gives the concluding remarks. 


\section{RELATED WORK}

As a popular approach to analyze the capacity region of multi-hop wireless networks, the MCF problem under the protocol interference model is however NP-hard in general [3]. A rigorous NP-hardness proof is given in [10]. A lower bound and upper bound of such an NP-hard problem can be computed by solving a linear programming (LP) problem under constraints based on the maximal independent sets and the maximal cliques [3], respectively. Searching for all the maximal independent sets or maximal cliques is still NP-hard. Even given all the maximal independent sets or maximal cliques, the LP MCF problem has to be solved with exponentially many constraints to generate tight capacity bounds. Considering the high computational complexity, there are many efforts in developing polynomial approximation algorithms [6], [10], [11] for the MCF problem over wireless networks.

In this paper, our focus is not on approximation algorithms. Instead, we study how to enhance the MCF formulation and extend the conflict-graph based constraints to generate the capacity region, particularly an achievable lower bound, over the CSMA/CA MAC. Although many MCF capacity studies [3], [10], [11] claim that the impact of 802.11 is considered, it is in fact treated as a specific protocol interference model to define the conflict graph for centralized scheduling. The fundamental contribution of this paper is to derive necessary and sufficient conditions for MCF flow allocation, which take the CSMA/CA randomness nature into account. Note that the advance in the polynomial approximation algorithms could be exploited to improve the efficiency in solving our model.

\section{Optimal Operation Point}

We have developed a protocol-independent analysis in [8], revealing that the family of CSMA/CA protocols share the same optimal operation point where the maximum protocol capacity is achieved. The protocol-independent analysis is inspired by the concept of virtual slot [12], [13]. Observed at the timescale of virtual slot, all the CSMA/CA protocols show the same behavior that the channel alternates among the statuses of idling, successful transmission, and collision. Moreover, we have proved that the traffic arrival process at each virtual slot can be accurately described or well approximated by a Poisson random variable, when channel accesses from different users are independent (e.g., in 802.11 DCF) or weakly correlated (e.g., in 802.15.4 contention access period) [8]. If the size variation of different types of virtual slots were ignored, the virtual-slot system would behave stochastically similar to a slotted ALOHA protocol. Therefore, the wellknown S-G analysis for slotted ALOHA can be extended into a virtual slot based $S-G$ analysis (VS S-G).

Let $G$ denote the Poisson traffic load in a slot. The probability of seeing an idle (a successful transmission) slot $p_{d}\left(p_{s}\right)$ equals the probability that zero (a single) transmission trail happens at a slot. That is, $p_{d}=e^{-G}, p_{s}=G e^{-G}$, and the probability of seeing a collision slot $p_{c}=1-G e^{-G}-e^{-G}$. Let $\sigma, T_{s}$, and $T_{c}$ denote the lengths of virtual slots associated with channel idling, successful transmission, and collisions, respectively. The steady-state channel utilization $R_{s}$ can be computed as

$$
R_{s}=\frac{p_{s} T_{s}}{p_{d} \sigma+p_{s} T_{s}+p_{c} T_{c}}
$$

To obtain the maximum channel utilization (equivalently, the maximum MAC protocol capacity), we can determine the optimal workload $G^{*}$ according to $\left.\frac{d}{d G} R_{s}\right|_{G=G^{*}}=0$. Let $p_{d}^{*}$, $p_{s}^{*}$, and $p_{c}^{*}$ denote the values of the probabilities under the optimal workload $G^{*}$. The maximum channel utilization can be obtained as $R_{s}^{*}=\frac{p_{s}^{*} T_{s}}{p_{d}^{*} \sigma+p_{s}^{*} T_{s}+p_{c}^{*} T_{c}}$. Let $L_{p}$ denote the payload size of a packet and $C$ the spectrum bandwidth, the maximum normalized throughput $R_{g}^{*}$ can be computed as

$$
R_{g}^{*}=\frac{p_{s}^{*} L_{p}}{C\left(p_{d}^{*} \sigma+p_{s}^{*} T_{s}+p_{c}^{*} T_{c}\right)}=\frac{R_{s}^{*} t_{p}}{T_{s}}
$$

where $t_{p}=\frac{L_{p}}{C}$ represents the effective transmission time within each successful period. The maximum normalized throughput indicates the optimal operation point.

\section{MCF FORMULATION}

We consider a single-channel wireless backhaul network, represented as a directed graph $\mathcal{G}(\mathcal{N}, \mathcal{L})$ with node set $\mathcal{N}$ and link set $\mathcal{L}$. Each node, $n_{i}$, has a communication range $\chi_{i}$ and a potentially larger interference range $\chi_{i}^{\prime}$. There is a directed link $l_{i j}$ from node $n_{i}$ to $n_{j}$ if $d_{i j} \leq \chi_{i}$. The capacity of link $l_{i j}$ is denoted as $C_{i j}$ and assumed to be time invariant. Bidirectional transmissions between two nodes are described by two directed links, one for each direction. There are two models, the protocol interference model and the physical interference model [3], [9], to define the conditions for a successful transmission. We adopt the protocol interference model in this paper.

\section{A. Basic MCF Formulation}

Let $(\nu, \eta)$ denote an ingress/egress pair of the backhaul network and $\Lambda$ the set of ingress/egress pairs. We further use $x_{\nu \eta}(i, j)$ to denote the portion of $n_{\nu}$-to- $n_{\eta}$ traffic flow that traverses the link $l_{i j}$. The classic MCF formulation without considering the wireless interference is expressed as

$$
\max \sum_{(\nu, \eta)} \sum_{l_{\nu i} \in \mathcal{L}} x_{\nu \eta}(\nu, i)
$$

subject to:

$$
\begin{aligned}
& \sum_{l_{j i} \in \mathcal{L}} x_{\nu \eta}(j, i)=\sum_{l_{i j} \in \mathcal{L}} x_{\nu \eta}(i, j), \\
& \forall(\nu, \eta) \in \Lambda \text { and } n_{i} \in \mathcal{N} /\left\{n_{\nu}, n_{\eta}\right\} \\
& \sum_{l_{i \nu} \in \mathcal{L}} x_{\nu \eta}(i, \nu)=0, \quad \forall(\nu, \eta) \in \Lambda \\
& \sum_{l_{\eta i} \in \mathcal{L}} x_{\nu \eta}(\eta, i)=0, \quad \forall(\nu, \eta) \in \Lambda \\
& \sum_{(\nu, \eta)} x_{\nu \eta}(i, j) \leq C_{i j}, \quad \forall l_{i j} \in \mathcal{L} \\
& x_{\nu \eta}(i, j) \geq 0, \quad \forall l_{i j} \in \mathcal{L} \text { and }(\nu, \eta) \in \Lambda .
\end{aligned}
$$


The formulation states that we aim at maximizing the aggregate throughput traversing all the ingress/egress pairs, with the five constraints [3] of flow conservation constraint (4), source constraint (5), destination constraint (6), link constraint (7), and nonnegative constraint (8).

\section{B. Conflict Graph Constraints}

In a conflict graph $\mathcal{F}$, the vertices correspond to the links in the network graph $\mathcal{G}$. According to the protocol interference model [3], we draw an edge in the conflict graph between vertices $l_{i j}$ and $l_{p q}$ if $d_{a b} \leq \chi_{a}^{\prime}$ for $a b=i q$, qi, $i p, p i, j p$, $p j, j q$ or $q j$. Such a conflict relation implies that a successful transmission over the CSMA/CA MAC (e.g., the 802.11 DCF) requires that both the sender and the receiver are free of interference from other nodes. Let $\mathcal{I}_{1}, \mathcal{I}_{2}, \cdots, \mathcal{I}_{K}$ denote all the $K$ maximal independent sets in $\mathcal{F}$, and $\alpha_{u}\left(0 \leq \alpha_{u} \leq 1\right)$ denote the fraction of time allocated to the independent set $\mathcal{I}_{u}$, $u=1, \cdots, K$. We can then add the constraints given in (9) and (10) to the basic MCF formulation to obtain the maximum throughput of a wireless backhaul network.

$$
\begin{aligned}
& \sum_{u=1}^{K} \alpha_{u} \leq 1 \\
& \sum_{(\nu, \eta)} x_{\nu \eta}(i, j) \leq \sum_{u: l_{i j} \in \mathcal{I}_{u}} \alpha_{u} C_{i j}, \quad \forall l_{i j} \in \mathcal{L} .
\end{aligned}
$$

The maximal independent set based solution gives a lower bound to the network capacity [3]. The capacity is achievable by the scheduling that each maximal independent set take turns to transmit, with the proportion of transmission time of each set specified by $\alpha_{u}$.

An upper bound of the maximum network throughput can be solved by finding all the maximal cliques and augmenting the basic MCF formulation with the constraints that the total usage within each maximal clique is at most 1 [3]. Suppose that there are $M$ maximal cliques in the conflict graph $\mathcal{F}$, denoted as $\mathcal{C}_{1}, \cdots, \mathcal{C}_{M}$, respectively. If a link $l_{i j}$ within a maximal clique is allocated the transmission time of $t_{i j}$ during the whole clique transmission interval $T$, the clique constraint can be expressed in terms of the normalized throughput as

$$
\sum_{l_{i j} \in \mathcal{C}_{u}} \frac{1}{C_{i j}} \sum_{(\nu, \eta)} x_{\nu \eta}(i, j) \leq 1, \quad u=1, \cdots, M .
$$

\section{CAPACITY REgION OVER CSMA/CA}

In this section, we develop new methods to derive the upper bound and lower bound of the backhaul network capacity over the CSMA/CA MAC. We focus on the 802.11 DCF protocol due to its wide application in wireless backhaul networks.

\section{A. Upper Bound of the Capacity}

Let $\hat{X}$ denote the upper bound of the MCF throughput based on the clique constraint (11). We have the following theorem regarding a tighter upper bound of the network throughput over the CSMA/CA MAC.

Theorem 1: A tighter upper bound of the optimal throughput of a wireless mesh network based on a CSMA/CA MAC protocol is $\hat{F}=R_{g}^{*} \hat{X}$.

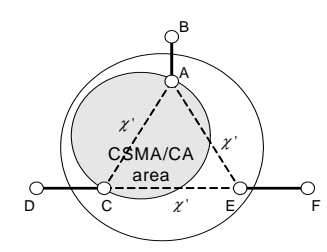

Fig. 1.

CSMA/CA area, CSMA/CA clique, and maximal clique.

Proof: The MCF formulation based on clique constraint of (11) gives an upper bound of the network throughput [3]. Within each maximal clique, we can concentrate each link into a virtual node contending for the channel access, since simultaneous transmissions will not be successful under the CSMA/CA protocol. Regarding such virtual nodes, the VS $\mathrm{S}-\mathrm{G}$ analysis in Section III determines that the maximum normalized throughput over a maximal clique is upper-bounded by $R_{g}^{*}$, when the distributed CSMA/CA MAC is applied.

We now apply the clique constraints with the maximum utilization of $R_{g}^{*}$ to the basic MCF formulation for $\max \sum_{(\nu, \eta)} \sum_{l_{\nu i} \in \mathcal{L}} f_{\nu \eta}(\nu, i)$. The flow is denoted by $f_{\nu \eta}(i, j)$ to emphasize that the formulation is to find the maximum throughput over the CSMA/CA, denoted by $\hat{F}$. Compared to the centralized scheduling case, the clique constraint under the CSMA/CA MAC is now expressed as

$$
\sum_{l_{i j} \in \mathcal{C}_{u}} \frac{1}{C_{i j}} \sum_{(\nu, \eta)} f_{\nu \eta}(i, j) \leq R_{g}^{*}, \quad u=1, \cdots, M .
$$

If we define $x_{\nu \eta}(i, j)=f_{\nu \eta}(i, j) / R_{g}^{*}$, the new MCF formulation with the constraint of (12) is then transformed back to the original clique-based MCF formulation assuming a centralized scheduling, associated with the constraint (11). Therefore, the optimal solutions $x_{\nu \eta}^{*}(i, j)=f_{\nu \eta}^{*}(i, j) / R_{g}^{*}$ and $\hat{F}=R_{g}^{*} \hat{X}$.

Obtaining a tight lower bound through clique-based MCF formulation is much more complex than computing the upper bound. We next study a new concept of CSMA/CA clique to facilitate the lower bound analysis. For simplification, in the remainder of this paper we assume that all the nodes have the same communication range $\chi$ and interference range $\chi^{\prime}(\geq \chi)$. The carrier sensing range equals the interference range.

\section{B. CSMA/CA Clique and CSMA/CA Area}

A circle with a diameter of $\chi^{\prime}$ is termed as a CSMA/CA area. Within a CSMA/CA area, all the nodes are within the carrier sensing range of each other, and the transmissions from different nodes are coordinated by the CSMA/CA protocol. It is obvious that a CSMA/CA area defines a clique, since any two links incident to nodes within the area conflict with each other. The clique is termed as the clique associated with the CSMA/CA area, or a CSMA/CA clique for convenience. Note that a CSMA/CA area may not be large enough to cover a maximal clique. An example is illustrated in Fig. 1, where links $l_{A B}, l_{C D}$, and $l_{E F}$ form a maximal clique due to the interference between node $n_{A}$ and $n_{C}, n_{A}$ and $n_{E}$, as well as $n_{C}$ and $n_{E}$. A CSMA/CA area in this scenario can at most cover two links, and the associated CSMA/CA clique is a subset of the maximal clique. 
1) Maximum Normalized Throughput: We have the following lemma regarding the maximum normalized throughput over a CSMA/CA area and the associated CSMA/CA clique.

Lemma 1: The total normalized throughput over a CSMA/CA clique can be transformed to that over the CSMA/CA area defining the clique, and vice versa. Thus, the maximum normalized throughput over a CSMA/CA clique is $R_{g}^{*}$.

Proof: Let $\mathcal{A}_{\mathcal{C}}$ denote the CSMA/CA area defining a clique $\mathcal{C}$. Let $n_{i} \in \mathcal{A}_{\mathcal{C}}$ denote that node $n_{i}$ is within the CSMA/CA area $\mathcal{A}_{\mathcal{C}}$. The clique $\mathcal{C}$ consists of links $\left\{l_{i j} \mid n_{i} \in \mathcal{A}_{\mathcal{C}}\right.$ or $n_{j} \in$ $\left.\mathcal{A}_{\mathcal{C}}\right\}$. Note that it is possible that one end, particularly, the sender node of a link belonging to the clique $\mathcal{C}$ is outside $\mathcal{A}_{\mathcal{C}}$, as shown in Fig. 2. Let $T$ denote the time interval for throughput measurement, and $t_{i j}$ the total transmission time occupied by link $l_{i j}\left(l_{i j} \in \mathcal{C}\right)$ during the time interval $T$. The total normalized throughput, $R_{g}$, over the clique $\mathcal{C}$ can be computed as

$$
\begin{aligned}
R_{g} & =\frac{1}{T} \sum_{l_{i j} \in \mathcal{C}} t_{i j}=\frac{1}{T}\left[\sum_{\substack{l_{i j} \in \mathcal{C} \\
n_{i} \in \mathcal{A}_{\mathcal{C}}, n_{j} \in \mathcal{A}_{\mathcal{C}}}} t_{i j}\right. \\
& \left.+\sum_{\substack{l_{i j} \in \mathcal{C} \\
n_{i} \in \mathcal{A}_{\mathcal{C}}, n_{j} \notin \mathcal{A}_{\mathcal{C}}}} t_{i j}+\sum_{\substack{l_{i j} \in \mathcal{C} \\
n_{i} \notin \mathcal{A}_{\mathcal{C}}, n_{j} \in \mathcal{A}_{\mathcal{C}}}} t_{i j}\right]
\end{aligned}
$$

Considering that in a clique only one successful transmission is allowed at a moment, the transmission time occupied by the sender node is equivalent occupied by the receiver node too. Moreover, a link $l_{i j} \in \mathcal{C}$ always has a corresponding link $l_{j i} \in \mathcal{C}$. Then, we can continue (13) with

$$
\begin{aligned}
& R_{g}=\frac{1}{T}\left[\sum_{\substack{l_{i j} \in \mathcal{C} \\
n_{i} \in \mathcal{A}_{\mathcal{C}}, n_{j} \in \mathcal{A}_{\mathcal{C}}}} t_{i j}+\sum_{\substack{l_{i j} \in \mathcal{C} \\
n_{i} \in \mathcal{A}_{\mathcal{C}}, n_{j} \notin \mathcal{A}_{\mathcal{C}}}}\left(t_{i j}+t_{j i}\right)\right] \\
= & \frac{1}{T} \sum_{l_{i j} \in \mathcal{C}, n_{i} \in \mathcal{A}_{\mathcal{C}}} t_{i j}^{\prime}=\frac{1}{T} \sum_{i: n_{i} \in \mathcal{A}_{\mathcal{C}}} \sum_{j: l_{i j} \in \mathcal{C}} t_{i j}^{\prime}=\sum_{i: n_{i} \in \mathcal{A}_{\mathcal{C}}} \frac{t_{n_{i}}}{T}
\end{aligned}
$$

where

$$
\begin{aligned}
t_{i j}^{\prime} & = \begin{cases}t_{i j} & \text { if } l_{i j} \in \mathcal{C}, n_{i} \in \mathcal{A}_{\mathcal{C}}, n_{j} \in \mathcal{A}_{\mathcal{C}} ; \\
t_{i j}+t_{j i} & \text { if } l_{i j} \in \mathcal{C}, n_{i} \in \mathcal{A}_{\mathcal{C}}, n_{j} \notin \mathcal{A}_{\mathcal{C}} .\end{cases} \\
t_{n_{i}} & =\sum_{j: l_{i j} \in \mathcal{C}} t_{i j}^{\prime}
\end{aligned}
$$

and $t_{n_{i}}$ represents the transmission time occupied by each node $n_{i} \in \mathcal{A}_{\mathcal{C}}$. The results of (15) and (16) show that the transmission time allocation in a CSMA/CA clique $\mathcal{C},\left\{t_{i j} \mid l_{i j} \in \mathcal{C}\right\}$ can be transformed to a transmission time allocation in the associated CSMA/CA area $\mathcal{A}_{\mathcal{C}},\left\{t_{n_{i}} \mid n_{i} \in \mathcal{A}_{\mathcal{C}}\right\}$. On the other hand, given a transmission allocation in $\mathcal{A}_{\mathcal{C}}$, if the destination node for each transmission is known too, the allocation then defines a transmission time allocation for the corresponding CSMA/CA clique $\mathcal{C}$. Such an equivalent relationship, in terms of total normalized throughput, between the CSMA/CA area $\mathcal{A}_{\mathcal{C}}$ and the associated clique $\mathcal{C}$ implies that the maximum throughput over a CSMA/CA clique equals $R_{g}^{*}$, under the CSMA/CA MAC protocol.

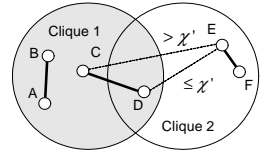

Fig. 3. Interference between two cliques.

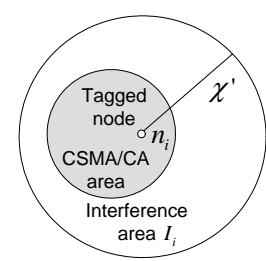

Fig. 4. Possible interference on a tagged node.

2) Achievability of Heterogeneous Rates: Although Lemma 1 indicates that the maximum throughput over a clique can be equivalently obtained by investigating a CSMA/CA area, the basic 802.11 DCF MAC assumes that each node is equipped with the same backoff schemes and thus grab a fair share of the capacity through channel contention [12]. However, for network dimensioning, the MCF solution normally allocates different rates to flows traversing a clique. Let $r_{i}$ denote the normalized rate of a sending node $n_{i}$. We have the following lemma regarding the heterogeneous rate allocation (the proof is ignored due to the limit of space).

Lemma 2: For 802.11 DCF MAC, any rate allocation over a single-hop CSMA/CA area $\mathcal{A}_{\mathcal{C}}$ satisfying

$$
\sum_{i: n_{i} \in \mathcal{A}_{\mathcal{C}}} r_{i} \leq R_{g}^{*}
$$

is achievable by differentiating the channel occupation time upon a successful transmission, i.e., the TXOP, for different sender nodes.

Based on the fact that TXOP differentiation is transparent to the backoff operation, we can prove another lemma.

Lemma 3: For 802.11 DCF MAC, a throughput $R_{g}$ achievable over a single-hop CSMA/CA area under homogeneous channel contention (where each node uses the same backoff parameters and TXOP) is also achievable under any specified heterogeneous rate allocation by applying the TXOP differentiation scheme.

\section{Lower Bound of the Capacity}

The fundamental reason that the clique-based MCF formulation gives an upper bound, which may not be achievable, of the network capacity over the CSMA/CA MAC is that the interference among the cliques in the multi-hop context can not be described by the clique constraint (12). Fig. 3 illustrate the interference between two cliques, where link $l_{A B}$ and link $l_{C D}$ belong to clique $\mathcal{C}_{1}$; link $l_{C D}$ and link $l_{E F}$ belong to clique $\mathcal{C}_{2}$. The interference between $l_{C D}$ and $l_{E F}$ is due to $d_{D E}<\chi^{\prime}$. Assume that at a certain moment, within clique $\mathcal{C}_{1}$ only node $n_{C}$ is trying to access the channel. However, if the link $l_{E F}$ in clique $\mathcal{C}_{2}$ is transmitting at this moment, node $n_{C}$ will not be able to utilize this transmission opportunity, although allowed in clique $\mathcal{C}_{1}$. Specifically, $n_{C}$ senses an idle channel and decides to transmit (because both nodes $n_{E}$ and $n_{F}$ are out of the carrier sensing range of $n_{C}$ ), but the packet delivered to node $n_{D}$ will be corrupted due to the transmission on link $l_{E F}$. Such an interference scenario illustrated in Fig. 3 is termed as hidden-node effect in the literature [14]. 
In order to obtain an achievable throughput, i.e., a lower bound, based on the clique model, the essence is to constraint the maximum throughput over each clique with the inter-clique interference taken into account. Define a uniform network as a network whose nodes are uniformly or regularly distributed in an area. We have the following lemma.

Lemma 4: In a uniform network, with the inter-clique interference considered, the total throughput over a CSMA/CA clique $R_{g}$ is achievable if $R_{g} \leq \frac{R_{g}^{*}}{4}$.

Proof: Lemma 1 tells that the total throughput over a CSMA/CA clique can be equivalent to that over the CSMA/CA area defining the clique. Lemma 3 further indicates that the total throughput over a CSMA/CA area can be conservatively evaluated by considering the homogeneous contention case, where each node contends with the same backoff parameters and same TXOP. Thus, the achievable throughput over a CSMA/CA clique is conservatively investigated here through studying the associated CSMA/CA area, with homogeneous channel contention from each node.

We consider a CSMA/CA area $\mathcal{A}_{\mathcal{C}}$, which defines the CSMA/CA clique $\mathcal{C}$. Note that a CSMA/CA area is covered by a circle with diameter $\chi^{\prime}$. Let $B\left(\mathcal{A}_{\mathcal{C}}\right)$ denotes the area of the circle. If the nodes are uniformly distributed with a density of $\rho$, the number of nodes contained by $\mathcal{A}_{\mathcal{C}}$ is $\rho B\left(\mathcal{A}_{\mathcal{C}}\right)$. When the CSMA/CA area $\mathcal{A}_{\mathcal{C}}$ is independently considered, with the same channel contention parameters, each node $n_{i} \in \mathcal{A}_{\mathcal{C}}$ can achieve the throughput of $R_{g}^{*} / \rho B\left(\mathcal{A}_{\mathcal{C}}\right)$ according to Lemma 2 .

To analyze the interference from other cliques on clique $\mathcal{C}$, let's consider a tagged sender node $n_{i} \in \mathcal{A}_{\mathcal{C}}$. It can be seen that all the nodes that may interfere with $n_{i}$ are within the circle $I_{i}$ centered at the tagged node with a radius of $\chi^{\prime}$, as shown in Fig. 4. A conservative condition to ensure the achievable throughput of $n_{i}$ under the worst-case interference is to consider the circle $I_{i}$ as one CSMA/CA area, which implies that any two nodes within $I_{i}$ can not transmit simultaneously. With homogeneous channel contention, a lower bound of the achievable throughput of the tagged node $n_{i}$ is $R_{g}^{*} / \rho B\left(I_{i}\right)$. Note that the transmissions outside the area $I_{i}$ can only lead to a higher throughput for node $n_{i}$. Specifically, the nodes within $I_{i}$ other than $n_{i}$ may be impacted by the nodes outside $I_{i}$, resulting in a smaller channel access probability within $I_{i}$. Nevertheless, the decrease of channel access from such nodes implies that the tagged node $n_{i}$ can grab the channel with a higher probability within $I_{i}$ and achieve a throughput higher.

In a uniform network ignoring the borderline effect, all nodes have the same stochastic behavior, and therefore the same achievable throughput, with homogeneous channel contention. Thus, the lower bound of the total achievable throughput over a CSMA/CA area, with inter-clique interference taken into account, is

$$
\widetilde{R_{g}}=\rho B\left(\mathcal{A}_{\mathcal{C}}\right) \cdot \frac{R_{g}^{*}}{\rho B\left(I_{i}\right)}=R_{g}^{*} \frac{\pi\left(\frac{\chi^{\prime}}{2}\right)^{2}}{\pi \chi^{\prime 2}}=\frac{R_{g}^{*}}{4}
$$

The lower bound of (18) ensures that $R_{g} \leq R_{g}^{*} / 4$ is achievable. The achievability under heterogeneous rate allocation is guaranteed by Lemma 3 .
Lemma 4 provides a sufficient condition regarding the achievable throughput over a CSMA/CA clique, under all the possible interference cases. Based on Lemma 4, we can establish the following theorem regarding the total throughput over the whole network (with proof ignored).

Theorem 2: For a uniform network, a lower bound of the optimal throughput over the CSMA/CA MAC is $\widetilde{F}=\frac{1}{4} \hat{F}$, where $\hat{F}$ is the upper bound given in Theorem 1. Thus, the lower bound is at least $\frac{1}{4}$ of the maximum capacity $F^{*}$, i.e., $\widetilde{F} \geq \frac{1}{4} F^{*}$.

\section{CONCLUSION}

This paper addresses the issue that the existing capacity studies of wireless backhaul networks, based on MCF formulation, assume impractical centralized scheduling and generate capacity region that is unachievable over a practical CSMA/CA MAC protocol. The fundamental contribution of this paper is to derive necessary and sufficient conditions for MCF flow allocation, which take into account the CSMA/CA randomness nature and therefore generate a tight upper bound and an achievable lower bound of the network capacity over the CSMA/CA MAC. For future work, we will have further indepth study on the capacity region over arbitrary networks and on polynomial algorithms to compute the achievable capacity region over the CSMA/CA MAC.

\section{REFERENCES}

[1] W. Wang, X. Liu, and D. Krishnaswamy, "Robust routing and scheduling in wireless mesh networks," in Proc. IEEE SECON, 2007, pp. 471-480.

[2] L. Badia, A. Erta, L. Lenzini, and M. Zorzi, "A general interferenceaware framework for joint routing and link scheduling in wireless mesh networks," IEEE Network, vol. 22, no. 1, pp. 32-38, Jan.-Feb. 2008.

[3] K. Jain, J. Padhye, V. Padmanabhan, and L. Qiu, "Impact of interference on multi-hop wireless network performance," in Proc. ACM MobiCom, 2003, pp. 66-80.

[4] T. Nandagopal, T.-E. Kim, X. Gao, and Vaduvur Bharghavan, "Achieving MAC layer fairness in wireless packet networks," in Proc. ACM MobiCom, 2000, pp. 87-98.

[5] K. N. Ramachandran, E. M. Belding, K. C. Almeroth, and M. M. Buddhikot, "Interference-aware channel assignment in multi-radio wireless mesh networks," in Proc. IEEE INFOCOM, 2006.

[6] V. S. Anil Kumar, M. V. Marathe, and S. Parthasarathy, "Algorithmic aspects of capacity in wireless neworks," in Proc. ACM SIGMETRICs, 2005, pp. 133-144.

[7] H. Viswanathan, S. Mukherjee, "Throughput-range tradeoff of wireless mesh backhaul networks," IEEE J. Sel. Areas Commun., vol. 24, no. 3, pp. 593-602, Mar. 2006.

[8] Y. Cheng, X. Ling, and W. Zhuang, "A protocol-independent approach for analyzing the optimal operation point of CSMA/CA protocols," in Proc. IEEE INFOCOM, Apr. 2009.

[9] P. Gupta and P. R. Kumar, "The capacity of wireless networks," IEEE Trans. Inform. Theory, vol. 46, no. 2, pp. 388-404, Mar. 2000.

[10] P.-J. Wan, "Multiflows in multihop wireless networks," in Proc. ACM MobiHoc, 2009.

[11] Y. Wang, W. Wang, X.-Y. Li, and W.-Z. Song, "Interference-aware joint routing and TDMA link scheduling for static wireless networks," IEEE Trans. Parallel Distrib. Syst., vol. 19, no. 2, pp. 1709-1726, Dec. 2008.

[12] G. Bianchi, "Performance analysis of the IEEE 802.11 distributed coordination function," IEEE J. Sel. Areas Commun., vol. 18, no. 3, pp. 535-547, Mar. 2000.

[13] Y. Cheng, X. Ling, W. Song, L.X. Cai, W. Zhuang, and X. Shen, "A cross-layer approach for WLAN voice capacity planning," IEEE J. Sel. Areas Commun., vol. 25, no. 4, pp. 678-688, May 2007.

[14] P. C. Ng and S. C. Liew, "Throughput analysis of IEEE 802.11 multihop Ad Hoc networks," IEEE/ACM Trans. Networking, vol. 15, no. 2 , pp. 309-322, Apr. 2007. 